\title{
Body mass index and long-term outcomes in patients with chronic total occlusions undergoing retrograde endovascular revascularization of the infra-inguinal lower limb arteries
}

\author{
Rafał Januszek ${ }^{1,2}$, Zoltan Ruzsa ${ }^{3,4}$, Andras Nyerges ${ }^{4}$, Viktor Óriás ${ }^{3}$, \\ Paweł Kleczyński ${ }^{1}$, Joanna Wojtasik-Bakalarz ${ }^{1}$, Artur Pawlik ${ }^{1}$, \\ Salech Arif ${ }^{1}$, Dariusz Dudek ${ }^{1,5}$, Stanisław Bartuśs ${ }^{1,5}$ \\ ${ }^{1} 2^{\text {nd }}$ Department of Cardiology and Cardiovascular Interventions, University Hospital, Krakow, Poland \\ ${ }^{2}$ Department of Clinical Rehabilitation, University of Physical Education, Krakow, Poland \\ ${ }^{3}$ Semmelweis University, Heart and Vascular Center, Cardiology Department, Budapest, Hungary \\ ${ }^{4}$ Bács-Kiskun County Hospital, Invasive Cardiology Department, \\ Teaching Hospital of Szent-Györgyi Albert Medical University, Kecskemét, Hungary \\ ${ }^{5} 2^{\text {nd }}$ Department of Cardiology, Jagiellonian University Medical College, Krakow, Poland
}

\begin{abstract}
Background: The aim of the present study is to assess the relationship between body mass index (BMI) and long-term clinical outcomes in retrograde endovascular recanalization (ER) regarding chronic total occlusions (CTOs) of the infra-inguinal lower limb arteries.

Methods: The study included patients who underwent retrograde ER of CTOs localized in superficial, popliteal or below-the-knee arteries. During follow-up, major adverse cardiac and cerebrovascular and major adverse lower limb events (MALE) were evaluated. MALE was defined as amputation, target lesion re-intervention, target vessel re-intervention and surgical treatment.

Results: The study included 405 patients at the mean age of $67.2 \pm 10.4$. The authors divided the overall group of patients according to BMI into $<25(n=156,38.5 \%)$ and $\geq 25 \mathrm{~kg} / \mathrm{m}^{2}(n=249$, $61.5 \%)$, and then into $<30(n=302,75.8 \%)$ and $\geq 30 \mathrm{~kg} / \mathrm{m}^{2}(n=103,24.2 \%)$. During the average follow-up 1,144.9 \pm 664.3 days, the mortality rate was higher in the group of patients with BMI $<25 \mathrm{~kg} / \mathrm{m}^{2}(10.5 \%$ vs. $5.3 \%, p=0.051)$, and in the group of patients with $B M I<30 \mathrm{~kg} / \mathrm{m}^{2}(8.7 \% \mathrm{vs} .2 .9 \%$, $p=0.048$ ). The comparison of Kaplan-Meier curves revealed borderline differences when assessing months to death for the BMI $<25 \mathrm{~kg} / \mathrm{m}^{2}(p=0.057)$ and BMI $<30 \mathrm{~kg} / \mathrm{m}^{2}(p=0.056)$ grouping variables. Conclusions: Obese and overweight patients undergoing CTO ER of the lower limb arteries from retrograde access are related to lower death rates during long-term follow-up. (Cardiol J 2021; 28, 4: 509-518)
\end{abstract}

Key words: lower limb atherosclerosis, chronic total occlusions, retrograde access, clinical outcomes, body mass index

\section{Introduction}

Intermittent claudication is becoming a more frequent symptom due to population aging and progress in the effective treatment of other dis- eases such as ischemic heart disease or respiratory disorders, which result in reduced exercise tolerance. This, consequently causes a more frequent clinical image of peripheral arterial disease (PAD) symptoms. Endovascular revascularization (ER)

Address for correspondence: Rafał Januszek, $\mathrm{MD}, \mathrm{PhD}, 2^{\text {nd }}$ Department of Cardiology and Cardiovascular Interventions, University Hospital in Krakow, ul. Kopernika 17, 31-501 Kraków, Poland, tel: +48 12424 71 70, fax: +48 124247180 , e-mail: jaanraf@interia.pl

Received: 14.02.2019 Accepted: 29.09.2019

This article is available in open access under Creative Common Attribution-Non-Commercial-No Derivatives 4.0 International (CC BY-NC-ND 4.0) license, allowing to download articles and share them with others as long as they credit the authors and the publisher, but without permission to change them in any way or use them commercially. 
is the treatment of choice in a majority of patients with symptomatic PAD. In the case of patients diagnosed with chronic total occlusion (CTO) of the lower limb arteries and failure of ER with antegrade access, retrograde access is a treatment option that can be offered prior to referring a patient to a vascular surgeon or possible optimal medical therapy. Several publications estimating long-term results of regular ER of PAD patients have been published $[1,2]$. The relationship between body mass index (BMI) and mortality rate in patients treated with percutaneous interventions has been widely investigated in patients with stable coronary artery disease (CAD), acute coronary syndromes and heart failure [3-6]. The relationship between $\mathrm{BMI}$ and mortality in patients with $\mathrm{PAD}$, especially those undergoing ER, is scarcely investigated and there are only a few publications available in this area [7-9].

Therefore, the aim of the present study is to evaluate the relationship between BMI and the long-term results of endovascular treatment from retrograde access in patients with PAD.

\section{Methods}

\section{Study population}

This study was planned as a prospective observational study of consecutive patients who underwent retrograde recanalization of CTO localized in the superficial femoral artery, popliteal artery or below-the-knee arteries. At two experienced and cooperating centers, all consecutive patients were enrolled after at least one unsuccessful antegrade recanalization of CTO who qualified for the retrograde approach. Each patient was also qualified for endovascular treatment after consultation by a vascular surgeon. The main factors disqualifying patients from surgical revascularization were anatomical reasons (lack of vessel circumference in the course of atherosclerosis), morphological (narrow and winding peripheral vessels) or poor clinical condition and high perioperative risk. Antegrade failure was defined as the inability to wire the distal part of the vessel behind the occlusion via the access site, located in the contralateral artery or proximal to the CTO lesion. The inability to wire the distal part of the CTO was both due to a failure to penetrate the lesion or because of a failure to return to the arterial lumen after subintimal recanalization in selected cases with suitable anatomy and morphology of target lesions. According to the local protocol, patients were screened for concomitant diseases, risk factors, and medication prior to the procedure. In all patients before the procedure, the ankle-brachial index was examined and severity of PAD was assessed according to Rutherford and/or Fontaine scale. The decision regarding retrograde recanalization and access site was based on prior angiography. The procedure of retrograde recanalization was performed under local anesthesia and required two access sites: antegrade and retrograde. Both, proximal and distal punctures were done under the guidance of vascular ultrasound and/or fluoroscopy. The choice of the type of antegrade access site was determined by many factors, including anatomical conditioning, type of vascular lesions (dissemination, calcifications, length), technical possibilities (having sufficiently long catheters), type of occlusions, its length and probability of blood flow restoration. For the proximal access site, the contralateral femoral artery was usually used, and 6 Fr vascular sheaths belonged to the most used. The distal access site was usually chosen in the reconnection area of the artery (needle: $12-15 \mathrm{~mm}, 21 \mathrm{G}$ ). In case of instability of distal puncture, 4 Fr vascular sheaths were needed to obtain support during the procedure. In a few cases, the distal access site was also used for the revascularization of more peripheral parts of the artery. The hydrophilic 0.035 " guidewire was used for the antegrade access site. Occlusions were crossed from the retrograde access site with a non-hydrophilic 0.018 " guidewire. After crossing the occlusion with a wire via the retrograde approach, pre-dilatation with a balloon catheter was performed. Stent implantation was based on the decision of the operator. After the procedure, the distal sheet was removed immediately, and the proximal one was maintained for up to $4 \mathrm{~h}$ when the femoral artery was punctured, which was conditioned by unfractionated heparin use. Due to dissection, in some cases, the balloon inflations were performed from both ante- and retrograde access (kissing balloon technique) to tear the dissection and facilitate capturing the wire with the diagnostic catheter. The first antegrade puncture site was used to visualize the vessel during the retrograde access procedure and was defined as the angiography first access site. In some patients, due to anatomical, morphological or technical problems, it was not possible to reach the distal part of the artery treated with retrograde access for angiography, and these patients had to undergo puncture of another artery which was defined as the second, third or fourth antegrade angiography access site. In periprocedural treatment, all patients received double antiplatelet therapy: acetylsalicylic acid 
$75 \mathrm{mg}$ - permanently, and clopidogrel $75 \mathrm{mg}$ for 3 months, a high dose of statin and according to a local protocol, low-molecular-weight heparin for 4 weeks. In the long-term follow-up, which lasted on average $1,144.9 \pm 664.3$ days, patients were evaluated for major adverse cardiac and cerebrovascular events (MACCE) as well as major adverse limb events (MALE). Data were collected between 2006 and 2016. In this paper, MACCE were predefined as death, stroke/transient ischemic attack, myocardial infarction, percutaneous coronary intervention (PCI) or coronary artery bypass grafting (CABG). MALE was defined as amputation, target lesion re-intervention, target vessel re-intervention and surgical treatment. Due to shortages in the available database, the level of amputation was not highlighted in the presented publication and alike high-, mid- and low-amputations, they were included in the term of overall amputation rate. The protocol complied with the Declaration of Helsinki, and all participants provided written informed consent before enrollment.

\section{Ethical approval and consent to participate}

All participants read the purpose statement of the investigation and signed informed consent. This study was approved by the local Research Ethics Committee and was therefore performed in accordance with the ethical standards of the 1964 Declaration of Helsinki and its later amendments.

\section{Statistical analysis}

Categorical variables are presented as numbers and percentages. Continuous variables are expressed as mean \pm standard deviation or median and interquartile range. Normality was assessed using the Shapiro-Wilk test. Equality of variance was assessed using Levene's test. Differences between groups were compared using the Student's or Welch's t-test depending on the equality of variances for normally distributed variables. The Mann-Whitney U test was applied in cases of continuous variables without normal distribution. Categorical variables were compared via the Pearson $\chi^{2}$ or the Fisher exact test if $20 \%$ of cells had a count less than 5 . Ordinal variables were compared with the Cochran-Armitage trend test. To analyze survival rate in selected risk groups, Kaplan-Meier curves were drawn. The log-rank statistic was used to test the differences in the outcome between groups. All statistical analyses were performed with JMP $®$, Version 13.1.0 (SAS Institute INC., Cary, NC, USA).

\section{Results}

\section{General characteristics}

In total, 405 patients were included in the study. There were $156(38.5 \%)$ patients with BMI < $<25 \mathrm{~kg} / \mathrm{m}^{2}$ and $302(74.6 \%)$ patients with BMI < $<30 \mathrm{~kg} / \mathrm{m}^{2}$. In both groups, patients with lower BMI were older when stratified for BMI $<25 \mathrm{~kg} / \mathrm{m}^{2}$ $(68.3 \pm 11.1$ vs. $66.51 \pm 9.8, \mathrm{p}=0.09)$ and also for BMI $<30 \mathrm{~kg} / \mathrm{m}^{2}(68.2 \pm 10.7$ vs. $64.4 \pm 8.9$, $\mathrm{p}=0.006)$, compared to patients with a higher BMI. Patients with higher BMI suffered from diabetes more often when stratified for BMI $\geq 25 \mathrm{~kg} / \mathrm{m}^{2}$ ( $35.9 \%$ vs. $59 \%, \mathrm{p}<0.0001)$ and for $\mathrm{BMI} \geq 30 \mathrm{~kg} / \mathrm{m}^{2}$ ( $43 \%$ vs. $70.9 \%, \mathrm{p}<0.0001$ ), as well as from CAD when classified for BMI $\geq 25 \mathrm{~kg} / \mathrm{m}^{2}(35.3 \%$ vs. $45.4 \%, \mathrm{p}=0.04)$, and for BMI $\geq 30 \mathrm{~kg} / \mathrm{m}^{2}(38.7 \%$ vs. $49.5 \%, \mathrm{p}=0.055)$. A general characterization of all groups assessed in the current study is presented in Table 1.

\section{Clinical presentation}

When stratified for BMI $<25 \mathrm{~kg} / \mathrm{m}^{2}$, the clinical stage of ischemic changes before angioplasty was more advanced in patients with lower BMI, both when using the Rutherford $(\mathrm{p}=0.04)$ and Fontaine classifications $(\mathrm{p}=0.01)$, however, when stratified for BMI $<30 \mathrm{~kg} / \mathrm{m}^{2}$, there were no significant differences in clinical presentation of ischemia before angioplasty between patients with lower and higher BMI ( $\mathrm{p}=0.6$ and $\mathrm{p}=0.96$ ). This is presented in Table 2.

\section{Angiography and procedural indices}

According to the Trans-Atlantic Inter Society Consensus (TASC II) classification, the authors did not observe any significant differences between patients with lower and higher mean BMI values when stratified for BMI $<25$ and $<30 \mathrm{~kg} / \mathrm{m}^{2}$. In general, lesions tended to occur longer in patients with lower BMI in both stratifications, and reached statistical significance for the common femoral artery when stratified for BMI $<25 \mathrm{~kg} / \mathrm{m}^{2}(13.4 \pm$ $\pm 21.8 \mathrm{~mm}$ vs. $8 \pm 13.8 \mathrm{~mm}, \mathrm{p}=0.04)$ and the popliteal artery when stratified for BMI $<30 \mathrm{~kg} / \mathrm{m}^{2}$ $(51.2 \pm 52.5 \mathrm{~mm}$ vs. $34.3 \pm 42.9 \mathrm{~mm}, \mathrm{p}=0.02)$. Angiographic indices are presented in Table 2.

\section{Clinical endpoints}

During the 120-month-long follow-up period, the authors did not observe significant differences in reintervention or amputation rates, the frequency of lower extremity bypass surgery and/ 
Table 1. General characteristics and clinical presentation.

\begin{tabular}{|c|c|c|c|c|c|c|}
\hline & \multicolumn{2}{|c|}{ Body mass index } & \multirow[t]{2}{*}{$\mathbf{P}$} & \multicolumn{2}{|c|}{ Body mass index } & \multirow[t]{2}{*}{$\mathbf{P}$} \\
\hline & $<25 \mathrm{~kg} / \mathrm{m}^{2}$ & $\geq 25 \mathrm{~kg} / \mathrm{m}^{2}$ & & $<30 \mathrm{~kg} / \mathrm{m}^{2}$ & $\geq 30 \mathrm{~kg} / \mathrm{m}^{2}$ & \\
\hline \multicolumn{7}{|l|}{ General characteristics } \\
\hline Age [years] & $68.3 \pm 11.1$ & $66.5 \pm 9.8$ & $0.09^{w}$ & $68.2 \pm 10.7$ & $64.4 \pm 8.9$ & $0.006^{u}$ \\
\hline Gender, males & $91(58.3 \%)$ & $162(65.1 \%)$ & $0.17^{P}$ & $192(63.6 \%)$ & $61(59.2 \%)$ & $0.43^{P}$ \\
\hline COPD & $22(14.1 \%)$ & $21(8.4 \%)$ & $0.07^{P}$ & $35(11.6 \%)$ & $8(7.8 \%)$ & $0.27^{P}$ \\
\hline Hypertension & $141(90.4 \%)$ & $232(93.2 \%)$ & $0.31^{P}$ & $274(90.7 \%)$ & $99(96.1 \%)$ & $0.08^{P}$ \\
\hline Diabetes & $56(35.9 \%)$ & $147(59 \%)$ & $<0.0001^{\mathrm{P}}$ & $130(43 \%)$ & $73(70.9 \%)$ & $<0.0001^{\mathrm{P}}$ \\
\hline Renal failure & $23(14.7 \%)$ & $44(17.7 \%)$ & $0.44^{p}$ & $46(15.2 \%)$ & $21(20.4 \%)$ & $0.22^{P}$ \\
\hline CAD & $55(35.3 \%)$ & $113(45.4 \%)$ & $0.04^{P}$ & $117(38.7 \%)$ & $51(49.5 \%)$ & $0.055^{P}$ \\
\hline Hyperlipidemia & $15(88.2 \%)$ & $27(90 \%)$ & $1.0^{\mathrm{F}}$ & $30(88.2 \%)$ & $12(92.3 \%)$ & $1.0^{\mathrm{F}}$ \\
\hline Stroke/TIA & $13(8.3 \%)$ & $12(4.8 \%)$ & $0.15^{P}$ & $22(7.3 \%)$ & $3(2.9 \%)$ & $0.11^{P}$ \\
\hline Smoking & $57(55.3 \%)$ & $68(50 \%)$ & $0.41^{P}$ & $92(50.5 \%)$ & $33(57.9 \%)$ & $0.33^{P}$ \\
\hline Prior PTA & $36(23.1 \%)$ & $59(23.7 \%)$ & $0.88^{P}$ & $67(22.2 \%)$ & $28(27.2 \%)$ & $0.3^{\mathrm{P}}$ \\
\hline Prior bypass & $36(23.1 \%)$ & $59(23.7 \%)$ & $0.63^{P}$ & $33(10.9 \%)$ & $10(9.7 \%)$ & $0.72^{P}$ \\
\hline \multicolumn{7}{|c|}{ Clinical presentation before angioplasty } \\
\hline Rutherford classification: & & & $0.04^{\mathrm{CA}}$ & & & $0.6^{\mathrm{CA}}$ \\
\hline 0 & $0(0 \%)$ & $0(0 \%)$ & & $0(0 \%)$ & $0(0 \%)$ & \\
\hline 1 & $13(8.3 \%)$ & $18(7.2 \%)$ & & $26(8.6 \%)$ & $5(4.8 \%)$ & \\
\hline 2 & $16(10.3 \%)$ & $43(17.3 \%)$ & & $46(15.2 \%)$ & $13(12.6 \%)$ & \\
\hline 3 & $18(11.5 \%)$ & $47(18.9 \%)$ & & $43(14.2 \%)$ & $22(21.4 \%)$ & \\
\hline 4 & $36(23.1 \%)$ & $52(20.9 \%)$ & & $66(21.8 \%)$ & $22(21.4 \%)$ & \\
\hline 5 & $28(17.9 \%)$ & $29(11.6 \%)$ & & $43(14.2 \%)$ & $14(13.6 \%)$ & \\
\hline 6 & $45(28.8 \%)$ & $60(24.1 \%)$ & & $78(25.8 \%)$ & $27(26.2 \%)$ & \\
\hline Fontaine scale: & & & $0.01^{\mathrm{CA}}$ & & & $0.96^{\mathrm{CA}}$ \\
\hline $2 a$ & $16(10.3 \%)$ & $21(8.4 \%)$ & & $32(10.6 \%)$ & $5(4.8 \%)$ & \\
\hline $2 b$ & $33(21.1 \%)$ & $95(38.1 \%)$ & & $90(29.8 \%)$ & $38(36.9 \%)$ & \\
\hline 3 & $34(21.8 \%)$ & 47 (18.9\%) & & $60(19.9 \%)$ & $21(20.4 \%)$ & \\
\hline 4 & 73 (46.8\%) & $86(34.5 \%)$ & & $120(39.7 \%)$ & $39(37.9 \%)$ & \\
\hline
\end{tabular}

CA - Cochran-Armitage test for trend; CAD — coronary artery disease; F — Fisher's exact test; P — Pearson's chi-squared test; $\mathrm{S}$ - Student's t-test; U - Mann-Whitney U test; PTA — percutaneous transluminal angioplasty; TIA — transient ischemic attack

or stroke as well as transient ischemic attack rates between patients with higher and lower BMI when stratified for $<25 \mathrm{~kg} / \mathrm{m}^{2}$ and $<30 \mathrm{~kg} / \mathrm{m}^{2}$. Nonetheless, death rate at the end of the followup period was higher in patients with lower BMI when stratified for BMI $<25 \mathrm{~kg} / \mathrm{m}^{2}(10.5 \%$ vs. $5.3 \%, \mathrm{p}=0.051)$ and for BMI $<30 \mathrm{~kg} / \mathrm{m}^{2}(8.7 \%$ vs. $2.9 \%, \mathrm{p}=0.048)$. This is presented in Table 3 . However, the long-rank test considered months to death for grouping variable BMI $<25 \mathrm{~kg} / \mathrm{m}^{2}$ revealed higher death rates in the group of patients with lower BMI, but without statistical significance $(\mathrm{p}=0.057)$. Also, analysis for grouping variable $\mathrm{BMI}<30 \mathrm{~kg} / \mathrm{m}^{2}$ demonstrated a significant ten- dency without statistical significance $(\mathrm{p}=0.056)$. This is presented in Figure $1 \mathrm{~A}$ and $\mathrm{B}$ as well as Table 4 . The long-rank test reflecting months to death for grouping variable BMI did not reveal significant differences $(\mathrm{p}=0.26)$ after division into six subgroups (underweight: $\mathrm{BMI}<18 \mathrm{~kg} / \mathrm{m}^{2}$, normal-weight: $18 \mathrm{~kg} / \mathrm{m}^{2} \leq \mathrm{BMI}<25 \mathrm{~kg} / \mathrm{m}^{2}$; overweight: $25 \mathrm{~kg} / \mathrm{m}^{2} \leq \mathrm{BMI}<30 \mathrm{~kg} / \mathrm{m}^{2}$; class 1 obesity: $30 \mathrm{~kg} / \mathrm{m}^{2} \leq \mathrm{BMI}<35 \mathrm{~kg} / \mathrm{m}^{2}$; class 2 obesity: $35 \mathrm{~kg} / \mathrm{m}^{2} \leq \mathrm{BMI}<40 \mathrm{~kg} / \mathrm{m}^{2}$; class 3 obesity: BMI $\geq 40 \mathrm{~kg} / \mathrm{m}^{2}$ ). Nevertheless, during the first 12 months of follow-up, mortality rate was highest in the subgroup of underweight and class 3 obesity patients. This is presented in Table 5 and Figure 2 . 
Table 2. Procedural indices: angiography, culprit artery and lesion.

\begin{tabular}{|c|c|c|c|c|c|c|}
\hline & \multicolumn{2}{|c|}{ Body mass index } & \multirow[t]{2}{*}{$\mathbf{P}$} & \multicolumn{2}{|c|}{ Body mass index } & \multirow[t]{2}{*}{$\mathbf{P}$} \\
\hline & $<25 \mathrm{~kg} / \mathrm{m}^{2}$ & $\geq 25 \mathrm{~kg} / \mathrm{m}^{2}$ & & $<30 \mathrm{~kg} / \mathrm{m}^{2}$ & $\geq 30 \mathrm{~kg} / \mathrm{m}^{2}$ & \\
\hline TASC II: & & & $0.25^{\mathrm{CA}}$ & & & $0.76^{\mathrm{CA}}$ \\
\hline A & $23(16.8 \%)$ & $41(19 \%)$ & & $53(20.1 \%)$ & $11(12.4 \%)$ & \\
\hline B & $23(16.8 \%)$ & $56(25.9 \%)$ & & $52(19.7 \%)$ & $27(30.3 \%)$ & \\
\hline C & $36(26.3 \%)$ & $34(15.7 \%)$ & & $55(20.8 \%)$ & $15(16.8 \%)$ & \\
\hline $\mathrm{D}$ & $55(40.1 \%)$ & $39(37.9 \%)$ & & $104(39.4 \%)$ & $36(40.4 \%)$ & \\
\hline Treated leg: & & & $0.48^{P}$ & & & $0.3^{P}$ \\
\hline Left & $73(46.8 \%)$ & $126(50.6 \%)$ & & $149(49.3 \%)$ & $50(48.5 \%)$ & \\
\hline Left, right & $4(2.6 \%)$ & $10(4 \%)$ & & $8(2.6 \%)$ & $6(5.8 \%)$ & \\
\hline Right & $79(50.6 \%)$ & $113(45.4 \%)$ & & $145(48 \%)$ & $47(45.6 \%)$ & \\
\hline Angiography first access site: & & & $0.32^{P}$ & & & $0.18^{P}$ \\
\hline Brachial & $15(10.6 \%)$ & $15(6.8 \%)$ & & $24(8.9 \%)$ & $6(6.4 \%)$ & \\
\hline Femoral & $73(51.4 \%)$ & $128(57.7 \%)$ & & $142(52.4 \%)$ & $59(63.4 \%)$ & \\
\hline Radial & $54(38 \%)$ & $79(35.6 \%)$ & & $105(38.7 \%)$ & $28(30.1 \%)$ & \\
\hline \multicolumn{2}{|c|}{ Angiography number of access sites: } & & $0.58^{\mathrm{CA}}$ & & & $0.23^{\mathrm{CA}}$ \\
\hline 1 & $116(81.7 \%)$ & $178(79.5 \%)$ & & $214(78.7 \%)$ & $80(85.1 \%)$ & \\
\hline 2 & $23(16.2 \%)$ & $42(18.7 \%)$ & & $52(19.1 \%)$ & $13(13.8 \%)$ & \\
\hline 3 & $3(2.1 \%)$ & $2(0.9 \%)$ & & $5(1.8 \%)$ & $0(0 \%)$ & \\
\hline 4 & $0(0 \%)$ & $2(0.9 \%)$ & & $1(0.4 \%)$ & $1(1.1 \%)$ & \\
\hline Angiography first access side: & & & $0.8^{P}$ & & & $0.15^{P}$ \\
\hline Right & $100(70.4 \%)$ & $155(69.2 \%)$ & & $191(70.2 \%)$ & $64(68.1 \%)$ & \\
\hline Left & $42(29.6 \%)$ & $69(30.8 \%)$ & & $81(29.8 \%)$ & $30(31.9 \%)$ & \\
\hline Iliac artery — SL & $35(24.6 \%)$ & $42(18.9 \%)$ & $0.19^{P}$ & $58(21.2 \%)$ & $19(20.9 \%)$ & $0.94^{P}$ \\
\hline Deep femoral artery - SL & $12(8.4 \%)$ & $9(4.1 \%)$ & $0.08^{P}$ & $19(7 \%)$ & $2(2.2 \%)$ & $0.09^{P}$ \\
\hline \multicolumn{7}{|l|}{ Common femoral artery: } \\
\hline Tortuosity: slight & $9(7.9 \%)$ & $9(5.5 \%)$ & $0.43^{P}$ & $14(6.7 \%)$ & $4(5.9 \%)$ & $1.0^{\mathrm{F}}$ \\
\hline Chronic total occlusion & $10(8.7 \%)$ & $4(2.4 \%)$ & $0.02^{P}$ & $11(5.2 \%)$ & $3(4.4 \%)$ & $1.0^{\mathrm{F}}$ \\
\hline \multicolumn{7}{|l|}{ Calcification: } \\
\hline slight & $23(20.2 \%)$ & $34(20.9 \%)$ & $0.11^{\mathrm{CA}}$ & $46(22 \%)$ & $11(16.2 \%)$ & $0.2^{\mathrm{CA}}$ \\
\hline severe & $11(9.6 \%)$ & $6(3.7 \%)$ & & $14(6.70 \%)$ & $3(4.4 \%)$ & \\
\hline Lesion length [mm] & $13.4 \pm 21.8$ & $8 \pm 13.8$ & $0.04^{u}$ & $10.7 \pm 18.5$ & $8.6 \pm 15.1$ & $0.42^{\mathrm{U}}$ \\
\hline \multicolumn{7}{|l|}{ Superficial femoral artery: } \\
\hline \multicolumn{7}{|l|}{ Tortuosity: } \\
\hline slight & $52(38 \%)$ & $67(31.9 \%)$ & $0.42^{\mathrm{CA}}$ & $91(34.9 \%)$ & $28(32.6 \%)$ & $0.65^{\mathrm{CA}}$ \\
\hline severe & $3(2.2 \%)$ & $6(2.9 \%)$ & & $7(2.7 \%)$ & $2(2.3 \%)$ & \\
\hline Chronic total occlusion & $63(45 \%)$ & $103(47.7 \%)$ & $0.61^{P}$ & $122(45.9 \%)$ & $44(48.9 \%)$ & $0.61^{\mathrm{P}}$ \\
\hline \multicolumn{7}{|l|}{ Calcification: } \\
\hline extreme & $1(0.73 \%)$ & $1(0.5 \%)$ & $0.65^{\mathrm{CA}}$ & $2(0.8 \%)$ & $0(0 \%)$ & $0.46^{\mathrm{CA}}$ \\
\hline severe & $30(21.9 \%)$ & $53(25.2 \%)$ & & $64(24.5 \%)$ & $19(22.1 \%)$ & \\
\hline slight & $59(43.1 \%)$ & $86(40.9 \%)$ & & $109(41.8 \%)$ & $36(41.9 \%)$ & \\
\hline Lesion length [mm] & $107 \pm 97$ & $74.8 \pm 36.3$ & $0.97^{U}$ & $106.9 \pm 98.6$ & $108.2 \pm 95.9$ & $0.92^{U}$ \\
\hline Popliteal artery — lesion length & $52.4 \pm 54.9$ & $43.6 \pm 47.7$ & $0.24^{u}$ & $51.2 \pm 52.5$ & $34.3 \pm 42.9$ & $0.02^{U}$ \\
\hline Tibio-fibular trunk - SL & $41(28.9 \%)$ & $52(23.4 \%)$ & $0.24^{P}$ & $74(27.1 \%)$ & $19(20.9 \%)$ & $0.23^{P}$ \\
\hline Tibialis anterior artery - SL & $70(49.3 \%)$ & $105(47.3 \%)$ & $0.7^{P}$ & $133(48.7 \%)$ & $42(46.1 \%)$ & $0.67^{P}$ \\
\hline Peroneal artery - SL & $64(45.1 \%)$ & $79(35.6 \%)$ & $0.07^{P}$ & $112(41 \%)$ & $31(34.1 \%)$ & $0.23^{P}$ \\
\hline Tibialis posterior artery - SL & $86(60.6 \%)$ & $106(47.7 \%)$ & $0.01^{P}$ & $133(48.7 \%)$ & $42(46.1 \%)$ & $0.67^{P}$ \\
\hline Contrast volume [mL] & $106.5 \pm 67.8$ & $119.4 \pm 77.4$ & $0.12^{u}$ & $110.9 \pm 70.7$ & $124.5 \pm 82.4$ & $0.19^{u}$ \\
\hline Hospitalization time [days] & $5.44 \pm 4.6$ & $5 \pm 4.7$ & $0.11^{u}$ & $5.4 \pm 4.8$ & $4.7 \pm 4.3$ & $0.2^{\mathrm{U}}$ \\
\hline Intraprocedural complications & $4(2.8 \%)$ & $11(4.9 \%)$ & $0.32^{p}$ & $12(4.4 \%)$ & $3(3.2 \%)$ & $0.76^{F}$ \\
\hline Major vascular complications & $5(3.52 \%)$ & $5(2.2 \%)$ & $0.51^{F}$ & $9(3.3 \%)$ & $1(1.1 \%)$ & $0.46^{F}$ \\
\hline Minor vascular complications & $17(12 \%)$ & $26(11.6 \%)$ & $0.9^{P}$ & $33(12.1 \%)$ & $10(10.6 \%)$ & $0.7^{P}$ \\
\hline
\end{tabular}

CA — Cochran-Armitage test for trend; F — Fisher's exact test; P — Pearson's chi-squared test; SL — significant lesion; TASC — Trans-Atlantic Inter Society Consensus; U - Mann-Whitney U test 
Table 3. Study endpoints.

\begin{tabular}{|c|c|c|c|c|c|c|}
\hline & \multicolumn{2}{|c|}{ Body mass index } & \multirow[t]{2}{*}{$\mathbf{P}$} & \multicolumn{2}{|c|}{ Body mass index } & \multirow[t]{2}{*}{$\mathbf{P}$} \\
\hline & $<25 \mathrm{~kg} / \mathrm{m}^{2}$ & $\geq 25 \mathrm{~kg} / \mathrm{m}^{2}$ & & $<30 \mathrm{~kg} / \mathrm{m}^{2}$ & $\geq 30 \mathrm{~kg} / \mathrm{m}^{2}$ & \\
\hline Re-PTA & $47(30.72 \%)$ & $72(29.15 \%)$ & $0.73^{P}$ & $89(29.97 \%)$ & $30(29.13 \%)$ & $0.87^{P}$ \\
\hline Days to Re-PTA & $130.6 \pm 125.4$ & $196.9 \pm 339.6$ & $0.47^{\cup}$ & $151.3 \pm 202.2$ & $228.2 \pm 427.7$ & $0.07^{\cup}$ \\
\hline Stroke/TIA & $2(1.3 \%)$ & $5(2 \%)$ & $0.71^{\mathrm{F}}$ & $5(1.68 \%)$ & $2(1.94 \%)$ & $1^{\mathrm{F}}$ \\
\hline Days to stroke/TIA & $66.5 \pm 47.4$ & $130.6 \pm 113.9$ & $0.69^{u}$ & $150.8 \pm 92.1$ & $16 \pm 12.7$ & $0.052^{U}$ \\
\hline Death & $16(10.5 \%)$ & $13(5.3 \%)$ & $0.051^{P}$ & $26(8.7 \%)$ & $3(2.9 \%)$ & $0.048^{\mathrm{F}}$ \\
\hline Days to death & $249.4 \pm 360.7$ & $537.8 \pm 896.9$ & $0.86^{U}$ & $310.5 \pm 472.5$ & $969.7 \pm 1650.9$ & $0.69^{u}$ \\
\hline Amputation & $24(15.7 \%)$ & $30(12.4 \%)$ & $0.35^{P}$ & $38(12.8 \%)$ & $16(16.2 \%)$ & $0.4^{P}$ \\
\hline Days to amputation & $87.6 \pm 103.7$ & $60.9 \pm 87.4$ & $0.36^{u}$ & $76.1 \pm 102.1$ & $64.9 \pm 78$ & $0.84^{u}$ \\
\hline Lower extremity bypass & $6(4.3 \%)$ & $9(4.1 \%)$ & $0.93^{P}$ & $10(3.7 \%)$ & $5(5.6 \%)$ & $0.54^{F}$ \\
\hline Days to lower extremity bypass & $48.5 \pm 69.1$ & $95.3 \pm 90.8$ & $0.19^{u}$ & $128.8 \pm 91.2$ & $76.6 \pm 83.6$ & $0.15^{\mathrm{U}}$ \\
\hline
\end{tabular}

F - Fisher's exact test; P — Pearson's chi-squared test; PTA — percutaneous transluminal angioplasty; S — Student's t-test; TIA — transient ischemic attacks; $U$ - Mann-Whitney $U$ test

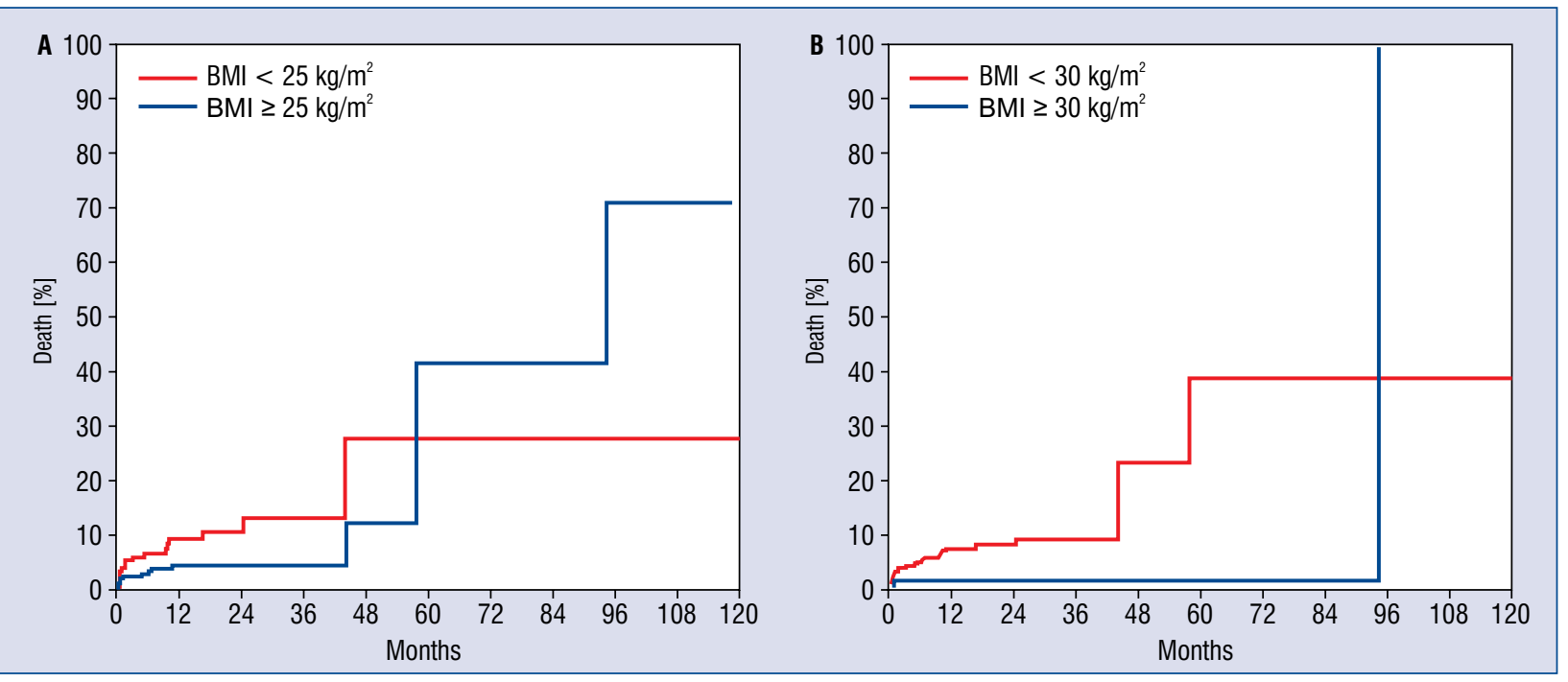

Figure 1. A. Months to death for grouping variable body mass index (BMI) $<25 \mathrm{~kg} / \mathrm{m}^{2}$. The log-rank test $\mathrm{p}$-value is equal to 0.057 ; B. Months to death for grouping variable $\mathrm{BMI}<30 \mathrm{~kg} / \mathrm{m}^{2}$. The log-rank test $\mathrm{p}$-value is equal to 0.056 .

\section{Clinical endpoints and COPD}

In the current study, 43 patients diagnosed with chronic obstructive pulmonary disease (COPD; 10.6\%), were divided according to BMI, similarly as in the overall group of patients. The authors compared clinical outcomes between patients with BMI $<25 \mathrm{~kg} / \mathrm{m}^{2}(51.2 \%)$ and $\geq 25 \mathrm{~kg} / \mathrm{m}^{2}$ (48.8\%) and between patients with BMI $<30 \mathrm{~kg} / \mathrm{m}^{2}$ $(81.4 \%)$ and $\geq 30 \mathrm{~kg} / \mathrm{m}^{2}$ (18.6\%). Particular clinical endpoints were higher in patients with lower BMI; however, they did not reach statistical significance when stratified for BMI $<25 \mathrm{~kg} / \mathrm{m}^{2}$ (reinterventions: $19.05 \%$ vs. $9.52 \%, \mathrm{p}=0.66$; deaths: $14.29 \%$ vs. $4.76 \%, \mathrm{p}=0.6$ and lower extremity bypass surgery: $21.05 \%$ vs. $5 \%, \mathrm{p}=0.18$ ) and for $\mathrm{BMI}<$ $<30 \mathrm{~kg} / \mathrm{m}^{2}$ (reinterventions: $17.65 \%$ vs. $0 \%$, $\mathrm{p}=0.57$; deaths: $11.76 \%$ vs. $0 \%, \mathrm{p}=0.57$; amputations: $14.29 \%$ vs. $12.5 \%, \mathrm{p}=1.0$; lower extremity bypass surgery: $15.63 \%$ vs. $0 \%, \mathrm{p}=0.56$ ).

\section{Discussion}

The main finding of the current study is that underweight, normal-weight and extremaly obese patients are at increased risk of death prior to endovascular treatment of CTOs of the lower 
Table 4. Cumulative risk of study endpoints according to body mass index (BMI) status.

\begin{tabular}{|c|c|c|c|c|c|c|c|c|c|c|c|}
\hline BMI & $0 \mathrm{M}$ & $12 \mathrm{M}$ & $24 M$ & $36 \mathrm{M}$ & $48 \mathrm{M}$ & $60 \mathrm{M}$ & $72 \mathrm{M}$ & $84 \mathrm{M}$ & $96 \mathrm{M}$ & $108 \mathrm{M}$ & $120 \mathrm{M}$ \\
\hline \multicolumn{12}{|l|}{$<25$} \\
\hline At risk & 153 & 92 & 35 & 10 & 5 & 3 & 3 & 3 & 3 & 3 & 2 \\
\hline CNE & 0 & 13 & 14 & 15 & 16 & 16 & 16 & 16 & 16 & 16 & 16 \\
\hline \multicolumn{12}{|l|}{$\geq 25$} \\
\hline At risk & 247 & 170 & 85 & 23 & 9 & 2 & 2 & 2 & 1 & 1 & - \\
\hline CNE & 0 & 10 & 10 & 10 & 11 & 12 & 12 & 12 & 13 & 13 & - \\
\hline \multicolumn{12}{|l|}{$<30$} \\
\hline At risk & 297 & 188 & 88 & 25 & 10 & 4 & 4 & 4 & 4 & 4 & 2 \\
\hline CNE & 0 & 21 & 22 & 23 & 25 & 26 & 26 & 26 & 26 & 26 & 26 \\
\hline \multicolumn{12}{|l|}{$\geq 30$} \\
\hline At risk & 103 & 74 & 32 & 8 & 4 & 1 & 1 & 1 & - & - & - \\
\hline CNE & 0 & 2 & 2 & 2 & 2 & 3 & 3 & 3 & - & - & - \\
\hline \multicolumn{12}{|l|}{ Total } \\
\hline At risk & 400 & 262 & 120 & 33 & 14 & 5 & 5 & 5 & 4 & 4 & 2 \\
\hline CNE & 0 & 23 & 24 & 25 & 27 & 28 & 28 & 28 & 29 & 29 & 29 \\
\hline
\end{tabular}

CNE - cumulative number of events, $\mathrm{M}-$ months

Table 5. Cumulative risk of death according to body mass index (BMI) in selected intervals.

\begin{tabular}{|c|c|c|c|c|c|c|c|c|c|c|c|}
\hline BMI & $\mathbf{O M}$ & $12 \mathrm{M}$ & $24 M$ & $36 \mathrm{M}$ & $48 M$ & $60 \mathrm{M}$ & $72 \mathrm{M}$ & $84 \mathrm{M}$ & $96 \mathrm{M}$ & $108 \mathrm{M}$ & $120 \mathrm{M}$ \\
\hline \multicolumn{12}{|l|}{$<18.5$} \\
\hline At risk & 8 & 2 & 1 & - & - & - & - & - & - & - & - \\
\hline CNE & 0 & 1 & 1 & - & - & - & - & - & - & - & - \\
\hline \multicolumn{12}{|c|}{$18.5 \leq \mathrm{BMI}<25$} \\
\hline At risk & 145 & 90 & 34 & 10 & 5 & 3 & 3 & 3 & 3 & 3 & 2 \\
\hline CNE & 0 & 12 & 13 & 14 & 15 & 15 & 15 & 15 & 15 & 15 & 15 \\
\hline \multicolumn{12}{|c|}{$25 \leq \mathrm{BMI}<30$} \\
\hline At risk & 144 & 96 & 53 & 15 & 5 & 1 & 1 & 1 & 1 & 1 & - \\
\hline CNE & 0 & 8 & 8 & 8 & 9 & 10 & 10 & 10 & 10 & 10 & - \\
\hline \multicolumn{12}{|c|}{$30 \leq \mathrm{BMI}<35$} \\
\hline At risk & 77 & 57 & 25 & 8 & 4 & 1 & 1 & 1 & - & - & - \\
\hline CNE & 0 & 1 & 1 & 1 & 1 & 2 & 2 & 2 & - & - & - \\
\hline \multicolumn{12}{|c|}{$35 \leq \mathrm{BMI}<40$} \\
\hline At risk & 15 & 12 & 6 & - & - & - & - & - & - & - & - \\
\hline CNE & 0 & 0 & 0 & - & - & - & - & - & - & - & - \\
\hline \multicolumn{12}{|l|}{$\mathrm{BMI} \geq 40$} \\
\hline At risk & 11 & 5 & 1 & - & - & - & - & - & - & - & - \\
\hline CNE & 0 & 1 & 1 & - & - & - & - & - & - & - & - \\
\hline
\end{tabular}

CNE - cumulative number of events; $\mathrm{M}$ - months

limb infra-inguinal arteries during the long-term follow-up period in comparison to the group of obese patients.

Several studies assessing the impact of BMI calculated before PCI have been published to date [3-5]. It was demonstrated that in the case of underweight, normal-weight and extremely obese patients with a $\mathrm{BMI} \geq 35 \mathrm{~kg} / \mathrm{m}^{2}$ present an increased death rate compared to other weights during the long-term follow-up period [3]. This 


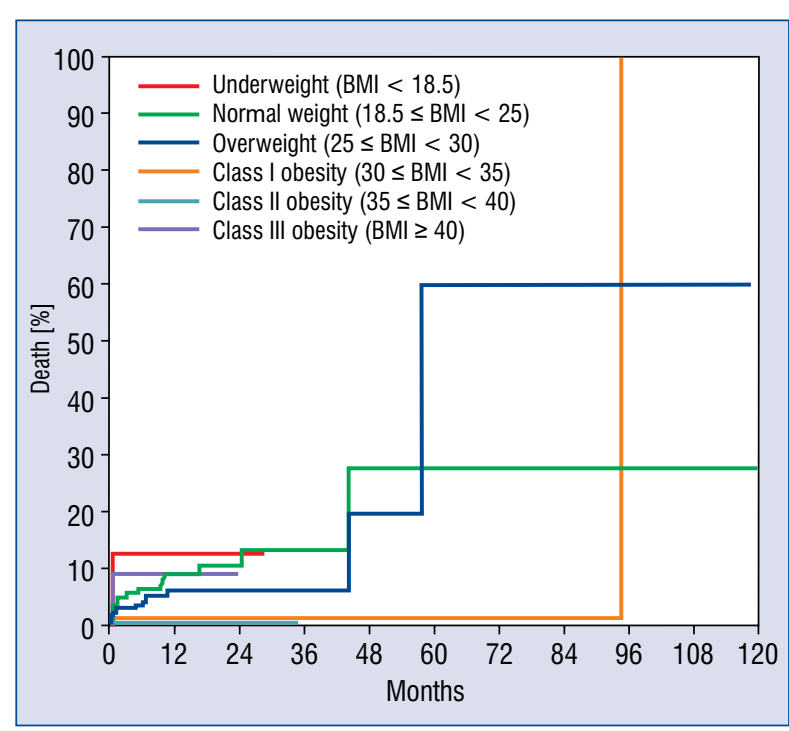

Figure 2. Months to death for grouping variable body mass index (BMI). The log-rank test $p$-value is equal to 0.26 .

correlation in patients with CAD treated using PCI has been described as the "obesity paradox". Several explanations and mechanisms have been attributed to this phenomenon. One of them is that obese patients are younger, which correlates to longer survival rate [3]. This relationship was also visible in the current analysis concerning patients with PAD. However, previously published studies combined the lower mean age with smaller rates of co-morbidities and less advanced atherosclerosis [3]. While some patterns of more advanced atherosclerosis like lesion length or the percentage of CTO lesions in patients with lower BMI were observed in the current study, the rate of co-morbidities such as diabetes and CAD was significantly higher in patients from higher BMI groups $\left(\geq 25 \mathrm{~kg} / \mathrm{m}^{2}\right.$ and $\left.\geq 30 \mathrm{~kg} / \mathrm{m}^{2}\right)$. Previously published studies included endocrine status modified by adipose tissue and the size of coronary vessels among the other factors possibly responsible for the protective effect of obesity in patients with CAD treated using PCI $[10,11]$. Obesity is widely recognized as a risk factor of atherosclerosis and related complications such as cardiovascular events and mortality. However, there are some data that do not confirm this relationship in patients with CAD [12]. Despite the fact that the World Health Organization endorses the use of BMI as the best measure for screening obesity, some studies demonstrated that it is not sufficient in assessing the amount of adipose tissue but is related to other indices such as waist/ /hip ratio [13, 14]. Bioelectrical impedance analysis and muscle handgrip strength measurement are among the best devices for estimating muscle mass and adipose tissue [15]. Another factor that may affect mortality in obese patients is better adherence to medical recommendations in the field of pharmacotherapy and prevention in this group of patients [16]. Antiplatelet and antithrombotic therapy related to bleeding complications was also found to have a potential relationship with clinical outcomes after PCI and CAD [17, 18].

The research related to the relationship between long-term survival and BMI in patients with PAD is very limited [7-9]. To our knowledge, this is the first study describing the relationship between BMI and clinical outcomes in patients with PAD treated using angioplasty from retrograde access. The study published by Kumakura et al. [7] including 652 patients with PAD confirmed the presence of the "obesity paradox" phenomenon in this group of patients. The authors indicated glomerular filtration rate, critical limb ischemia (CLI) and diabetes to be among other predictors of mortality in patients with PAD. The study published by Murata et al. [8] including 1,088 patients and comprising 1,306 limbs with critical ischemia treated with endovascular therapy confirmed the previously discovered relationship in patients with CAD treated using PCI. This study demonstrated that underweight patients with CLI are at increased risk of death during the median follow-up of 1.5 years compared to overweight and obese patients. Also, normal weight was associated with poorer survival rate during the follow-up compared to overweight and obese patients [8]. Furthermore, age, heart failure, aortic valve stenosis, renal failure, serum albumin, medication with anticoagulants and nonambulatory status were found to be negative predictors related to all-cause mortality [8]. Among the possible mechanisms, the authors suggest that malnutrition, related to low triglyceride level in patients with $\mathrm{PAD}$, may be related to increased risk of death [7]. One of the published studies attributed the "obesity paradox" phenomenon in patients with PAD to the presence of concomitant COPD [19]. A similar correlation in patients with COPD between BMI and mortality has been previously demonstrated [20]. The incidence of COPD in the study published by Galal et al. [19] almost reached $47 \%$, while in our study, it was only slightly above $10 \%$. The impact of COPD in the current study was too low to modify the general relationship between BMI and mortality. Moreover, among the subgroup of patients with COPD 
in the current study, the relationship between death rate and BMI was not significant during the follow-up period. Considering the discussions on the issue of a number of potential mechanisms contributing to the development of the so-called "obesity paradox" in patients with CAD and PAD treated with percutaneous interventions, the role of sarcopenia has been superficially discussed. It has been demonstrated in several studies that sarcopenia is associated with increased mortality $[21,22]$. The so-called U-shaped mortality curve relation to BMI could be owed to sarcopenia in underweight patients, while increased death rates in patients from severe and higher obesity classes may be related to the overwhelming influence of negative factors connected with obesity such as inflammatory processes or oxidative stress and lipid disorders [3].

In the case of patients with reduced body mass, muscle mass plays crucial role, and it has been demonstrated in the group of patients with COPD undergoing rehabilitation that both resistance training as well as endurance training have a positive effect on mortality [23]. For patients with $\mathrm{PAD}$ endurance training may be difficult to achieve due to limited walking, but any physical activity leading to increased muscle mass seems to be beneficial and should be recommended for every patient with PAD. In addition, every patient undergoing ER with PAD should be informed about the fact that underweight and lower body weight is associated with increased mortality in the follow-up period. It should be remembered that the risk of revascularization, even in patients with a significantly higher risk of death in the follow-up period due to low body weight at baseline, is lower in patients treated percutaneously than in surgery. Therefore, in the case of limb threat with amputation and the possibility of percutaneous treatment, it does not seem advisable to postpone the procedure in order to increase muscle mass and body weight in terms of improving prognosis.

\section{Limitations of the study}

Several limitations can be attributed to the current study. One of them is that the current group of patients was extracted from a group of 834 patients treated from retrograde access and was limited to 405 patients due to lack of BMI data, despite the fact that the BMI deficiencies were random and were not associated with any particular factor. This could have significantly affected the results.

\section{Conclusions}

The BMI value at baseline in patients with PAD undergoing ER of CTOs of the infra-inguinal lower limb arteries from retrograde access is associated with mortality during the follow-up period. Underweight and normal-weight persons are at increased risk of death after angioplasty when compared to obese individuals. Therefore, any physical activity leading to increased muscle mass seems to be beneficial and should be recommended to every PAD patient.

\section{Availability of data and materials}

The datasets generated and/or analyzed during the current study are available from the corresponding author upon reasonable request.

\section{Conflict of interest: None declared}

\section{References}

1. Ruzsa Z, Wojtasik-Bakalarz J, Nyerges A, et al. Long-Term follow-up after retrograde recanalization of superficial femoral artery chronic total occlusion. J Invasive Cardiol. 2017; 29(10): 336-339, indexed in Pubmed: 28974660.

2. Evans C, Peter N, Gibson M, et al. Five-year retrograde transpopliteal angioplasty results compared with antegrade angioplasty. Ann R Coll Surg Engl. 2010; 92(4): 347-352, doi: 10.130 8/003588410X12664192075099, indexed in Pubmed: 20501022.

3. Angerås O, Albertsson P, Karason K, et al. Evidence for obesity paradox in patients with acute coronary syndromes: a report from the Swedish Coronary Angiography and Angioplasty Registry. Eur Heart J. 2013; 34(5): 345-353, doi: 10.1093/eurheartj/ ehs217, indexed in Pubmed: 22947610.

4. Gruberg L, Weissman NJ, Waksman R, et al. The impact of obesity on the short-term and long-term outcomes after percutaneous coronary intervention: the obesity paradox? J Am Coll Cardiol. 2002; 39(4): 578-584, doi: 10.1016/s0735-1097(01)01802-2, indexed in Pubmed: 11849854.

5. Coutinho T, Goel K, Corrêa de Sá D, et al. Combining body mass index with measures of central obesity in the assessment of mortality in subjects with coronary disease: role of ,normal weight central obesity”. J Am Coll Cardiol. 2013; 61(5): 553-560, doi: 10.1016/j.jacc.2012.10.035, indexed in Pubmed: 23369419.

6. Shah R, Gayat E, Januzzi JL, et al. GREAT (Global Research on Acute Conditions Team) Network. Body mass index and mortality in acutely decompensated heart failure across the world: a global obesity paradox. J Am Coll Cardiol. 2014; 63(8): 778-785, doi: 10.1016/j.jacc.2013.09.072, indexed in Pubmed: 24315906.

7. Kumakura H, Kanai H, Aizaki M, et al. The influence of the obesity paradox and chronic kidney disease on long-term survival in a Japanese cohort with peripheral arterial disease. J Vasc Surg. 2010; 52(1): 110-117, doi: 10.1016/j.jvs.2010.02.008, indexed in Pubmed: 20478682.

8. Murata N, Soga Y, Iida O, et al. Complex relationship of body mass index with mortality in patients with critical limb ischemia 
undergoing endovascular treatment. Eur J Vasc Endovasc Surg. 2015; 49(3): 297-305, doi: 10.1016/j.ejvs.2014.10.014, indexed in Pubmed: 25524520.

9. Iida O, Soga Y, Hirano K, et al. Midterm outcomes and risk stratification after endovascular therapy for patients with critical limb ischaemia due to isolated below-the-knee lesions. Eur J Vasc Endovasc Surg. 2012; 43(3): 313-321, doi: 10.1016/j. ejvs.2011.11.025, indexed in Pubmed: 22240338.

10. O'Connor NJ, Morton JR, Birkmeyer JD, et al. Effect of coronary artery diameter in patients undergoing coronary bypass surgery. Northern New England Cardiovascular Disease Study Group. Circulation. 1996; 93(4): 652-655, doi: 10.1161/01.cir.93.4.652, indexed in Pubmed: 8640991.

11. Momin AU, Melikian N, Shah AM, et al. Leptin is an endothelialindependent vasodilator in humans with coronary artery disease: Evidence for tissue specificity of leptin resistance. Eur Heart J. 2006; 27(19): 2294-2299, doi: 10.1093/eurheartj/ehi831, indexed in Pubmed: 16543250.

12. Yusuf S, Hawken S, Ounpuu S, et al. INTERHEART Study Investigators. Effect of potentially modifiable risk factors associated with myocardial infarction in 52 countries (the INTERHEART study): case-control study. Lancet. 2004; 364(9438): 937-952, doi: 10.1016/S0140-6736(04)17018-9, indexed in Pubmed: 15364185.

13. World Health Organization. Obesity and Overweight. http://new. who.int/news-room/fact-sheets/detail/obesity-and-overweight (Accessed April 29, 2018).

14. Gelber RP, Gaziano JM, Orav EJ, et al. Measures of obesity and cardiovascular risk among men and women. J Am Coll Cardiol. 2008; 52(8): 605-615, doi: 10.1016/j.jacc.2008.03.066, indexed in Pubmed: 18702962.

15. Januszek R, Siudak Z, Dziewierz A, et al. Chronic obstructive pulmonary disease affects angiographic presentation and out- comes. Authors' reply. Pol Arch Intern Med. 2018; 128(3): 195 -196, doi: 10.20452/pamw.4237, indexed in Pubmed: 29600970.

16. Steinberg BA, Cannon CP, Hernandez AF, et al. Medical therapies and invasive treatments for coronary artery disease by body mass: the „obesity paradox” in the Get With The Guidelines database. Am J Cardiol. 2007; 100(9): 1331-1335, doi: 10.1016/j. amjcard.2007.06.019, indexed in Pubmed: 17950785.

17. Delhaye C, Wakabayashi K, Maluenda G, et al. Body mass index and bleeding complications after percutaneous coronary intervention: does bivalirudin make a difference? Am Heart J. 2010; 159(6): 1139-1146, doi: 10.1016/j.ahj.2010.03.011, indexed in Pubmed: 20569731.

18. Mak KH, Bhatt DL, Shao M, et al. The influence of body mass index on mortality and bleeding among patients with or at high-risk of atherothrombotic disease. Eur Heart J. 2009; 30(7): 857-865, doi: 10.1093/eurheartj/ehp037, indexed in Pubmed: 19233855.

19. Galal W, van Gestel YR, Hoeks SE, et al. The obesity paradox in patients with peripheral arterial disease. Chest. 2008; 134(5): 925-930, doi: 10.1378/chest.08-0418, indexed in Pubmed: 18641109.

20. Landbo C, Prescott E, Lange P, et al. Prognostic value of nutritional status in chronic obstructive pulmonary disease. Am J Respir Crit Care Med. 1999; 160(6): 1856-1861, doi: 10.1164/ ajrccm.160.6.9902115, indexed in Pubmed: 10588597.

21. Tang $\mathrm{T}, \mathrm{Wu} \mathrm{L}$, Yang $\mathrm{L}$, et al. A sarcopenia screening test predicts mortality in hospitalized older adults. Sci Rep. 2018; 8(1): 2923, doi: 10.1038/s41598-018-21237-9, indexed in Pubmed: 29440681.

22. Yang M, Hu X, Wang H, et al. Sarcopenia predicts readmission and mortality in elderly patients in acute care wards: a prospective study. J Cachexia Sarcopenia Muscle. 2017; 8(2): 251-258, doi: 10.1002/jcsm.12163, indexed in Pubmed: 27896949.

23. Iepsen UW, Jørgensen KJ, Ringbaek T, et al. A Systematic Review of Resistance Training Versus Endurance Training in COPD. J Cardiopulm Rehabil Prev. 2015; 35(3): 163-172, doi: 10.1097/ HCR.0000000000000105, indexed in Pubmed: 25692720. 